\title{
Metallurgical Analyses of Welding Using a Developed Mini-Robot
}

\author{
Oladebeye Dayo Hephzibah ${ }^{1,}$, Adejuyigbe Samuel Babatope ${ }^{2}$, Kareem Biliyaminu ${ }^{3}$ \\ ${ }^{1}$ Department of Mechanical Engineering Technology, Federal Polytechnic, Ado-Ekiti, Nigeria \\ ${ }^{2}$ Mechatronics Engineering Department, Federal University, Oye-Ekiti, Nigeria \\ ${ }^{3}$ Industrial and Production Engineering Department, Federal University of Technology, Akure, Nigeria
}

Email address:

dayobeye@yahoo.com (O. D. Hephzibah), solaakim73@gmail.com (O. D. Hephzibah)

${ }^{*}$ Corresponding author

\section{To cite this article:}

Oladebeye Dayo Hephzibah, Adejuyigbe Samuel Babatope, Kareem Biliyaminu. Metallurgical Analyses of Welding Using a Developed Mini-Robot. American Journal of Mechanical and Materials Engineering. Vol. 4, No. 2, 2020, pp. 26-36. doi: 10.11648/j.ajmme.20200402.12

Received: May 10, 2020; Accepted: May 25, 2020; Published: June 15, 2020

\begin{abstract}
This research work addressed the mechanical and microstructural properties of welded joints. The results show the minimum average hardness values as $133.83,102.13,103.42,95.15,96.78$ and 117.50 for various mini-robot welded mild steel plates of thickness $0.5 \mathrm{~mm}, 0.6 \mathrm{~mm}, 0.7 \mathrm{~mm}, 0.8 \mathrm{~mm}, 0.9 \mathrm{~mm}$ and $1.0 \mathrm{~mm}$, while the maximum average hardness values were as $145.67,119.08,113.28,106.58,113.42$ and 137.75 respectively. Results of the research have shown that the robot welding samples produced are high in hardness. This is responsible for low tensile stress values that may also mean low mini-robot welded sample extension. The robot welding samples developed gave low tensile strain values and this was expected because the robot welding samples developed had high hardness, low extension and low tensile stress. The microstructural study shows that the welded mini-robot samples had more fine structure than coarse (which is more pearlite than ferrite). The built welding robot has also provided a wide range of welding speeds from experimentation, significantly less welding time, wide weld length. The built welding robot has a range of welding time $(4.7-32.94 \mathrm{~s})$, welding speed starting at $4.41 \mathrm{~mm} / \mathrm{s}$ over the same range of 0.5-1.0 mm thicknesses for the mild steel plate and weld length. The thicker the mild steel plate, the lower the welding time and the higher the welding speed. This is valid when the built welding robot was used. The built welding robot worked very well and the results of Microstructural Analyses presented quality welds.
\end{abstract}

Keywords: Metallurgy, Robot Arc Welding, Microstructure, Tensile Strength, Brinell Hardness

\section{Introduction}

In today's manufacturing scenario it is critical to have good quality welding in order to produce a high quality product. In the welding field, welding quality depends primarily on the welding form, welding parameters and the mechanical properties of the welded metal and heat-affected zone (HAZ), which in turn is determined by the metallurgical characteristics and chemical compositions of the weld [1-5]. Gas arc welding (GMAW) is a welding method which connects metals to their melting points by heating them with an electric arc [6-10]. According to [11], GMAW is an adaptable method which can be used for welding a wide variety of metals including carbon steels, low alloy steels, stainless steels, aluminum alloys, magnesium, copper and copper alloys, and nickel alloys. It can also be used for welding sheet metal and relatively heavy parts, and can be used, as in this research, for semi-automatic welding or automated welding. [12] dealt with the effect of welding variables on the mechanical properties of a $10 \mathrm{~mm}$ thick low carbon steel plate welded using the Shielded Metal Arc Welding (SMAW) process. The soldering current, arc voltage, welding speed and electrode diameter were the parameters which were tested for welding. The welded samples were cut and machined to standard tensile, impact tightness, and speed requirements for the hardness test. The findings showed that the parameters chosen for welding had important effects on the mechanical properties of the welded samples. Increases in arc voltage and welding current have increased rigidity and reduced yield power, tensile strength and 
durability to effect. Increasing the welding speed from 40$66.67 \mathrm{~mm} / \mathrm{min}$ resulted in the welded samples having improved hardness characteristics.

According to [13], the effect of welding parameters (different type of electrode and current) on mechanical properties such as tensile strength, impact resistance and hardness of low carbon steel arc welded joints with SMAW were investigated. The results showed that the tensile strength, impact, and hardness on the welding metal have significant effects of welding variables (type of electrodes and current given). The result shows that the mechanical properties such as tensile strength, impact and hardness decrease for all types of electrodes as the amount of current provided increases. Most industries nowadays use robotic welding equipment for joining processes, particularly the automotive industries. The robotic welding process has more advantages than traditional manual processes, such as a more robust weld efficiency, higher process speed compared to traditional manual processes [14]. While robot welding is very simple, manual adjustment of the joining parameter must be performed. Proper parameters are important to the performance of the robot and to the efficiency of welding. Parameters of the welding process such as welding speed, welding current, shielding gas flow rate and gap distance play an important role in determining the mechanical properties of the weld such as tensile load [15]. The weld length in the proven robot welding (Automated) is clearly greater for the same steel plate specimen than in the electric arc welding (Manual). Welding velocity increases from all signs, on average as steel plate thickness increases. Nevertheless, the welding speed of the built-in welding robot is much greater than that of the electric arc welding device [16]. According to [17], the produced robot welding samples gave a pattern of comparatively lower modulus elasticity values than both the unwelded and the electric arc welding samples. This pattern was anticipated given the higher hardness values, lower extension values, tensile stress and tensile strain of the robot welding samples formed above those of the electric arc welding and unwelded (CONTROL) samples. [18], used robotic gas metal arc welding to test the effects of welding parameters on the butt joints. The butt joint was intended to define appropriate welding parameters for voltage welding, welding current and welding speed. The experiment involved using a low carbon steel specimen A1008 as base metal and AWS ER 70S-6 as the filler metal in the process of joining the ass. The joint has been tested to determine the tensile strength, which is defined as the weld's main characteristic, and also the weld's hardness is registered. The results showed that the highest tensile hardness of $239.05 \mathrm{MPa}(180 \mathrm{HV})$ was provided by a 24 volt welding voltage, 200-220 ampere current, and $45-$ $50 \mathrm{~cm} / \mathrm{min}$ speed. The results showed that the highest tensile hardness of $239.05 \mathrm{MPa}(180 \mathrm{HV})$ was provided by a 24 volt welding voltage, 200-220 ampere current, and $45-50 \mathrm{~cm} / \mathrm{min}$ speed.

To produce welded joints of low-alloy high-resistance steels with adequate mechanical properties and cracking resistance, high volume fraction of acicular ferrite must be obtained from welded metal. Acicular ferrite is one of the most frequently formed microstructural constituents in the welded metal deposits of low alloy steel and has a direct effect on mechanical properties, especially toughness and hardness [19-20].

\section{Instruments Deployed for the Experiments}

The following instruments as shown in figures 1 to 3 were deployed for carrying out experiments of mechanical properties and microstrucural analysis on the mini-robot welded mild steel plates of different thickness while the mini-robot shown in figure 4 was used to weld the mild steel plates samples.

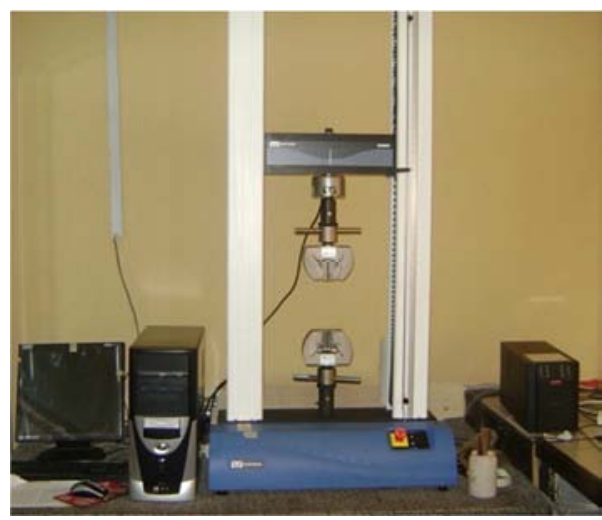

Figure 1. Universal Instron Machine, Model 3369, Maker (Instron).

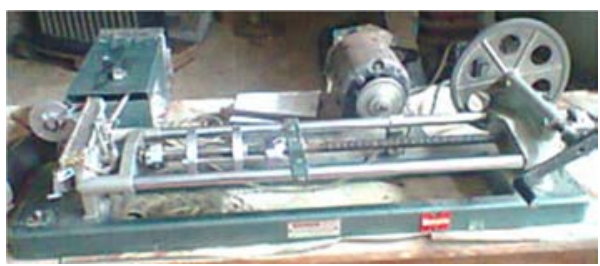

Figure 2. Brinell Hardness Testing Machine.

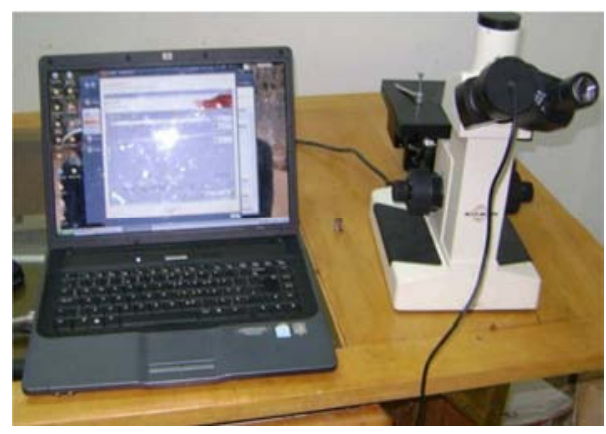

Figure 3. Accuscope Microscope with Camera (Serial no 0524011, Maker (Princeton, US).

\subsection{Developed Welding Mini-Robot}

The built welding robot can weld mild steel plates linearly along the length of the guide $470 \mathrm{~mm}$ on X-axis, $350 \mathrm{~mm}$ on $\mathrm{Y}$-axis and $110 \mathrm{~mm}$ on $\mathrm{Z}$-axis. 


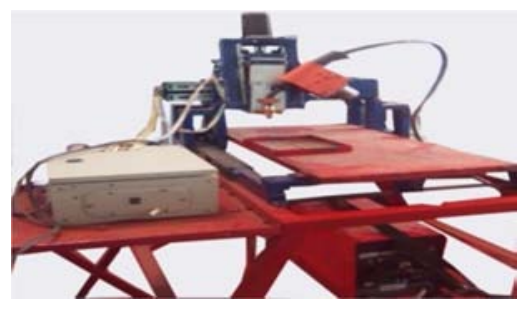

Figure 4. Assembly of the Developed Welding Robot (Front View).

\subsection{Specification of the Developed Welding Mini-robot}

The specification of the developed welding mini-robot is shown in Table 1.

Table 1. Specification of the developed welding mini-robot.

\begin{tabular}{ll}
\hline Specification & Cartesian type \\
\hline duty cycle Range & $(10 \%-60 \%)$ \\
welding current range & $(45 \mathrm{~A}-90 \mathrm{~A})$ \\
welding voltage range & $(16.25 \mathrm{~V}-18.5 \mathrm{~V})$ \\
electrode size & $0.8 \mathrm{~mm}$ \\
\hline
\end{tabular}

\section{Tensile Strength Test of Welded Mild Steel Plate with the Developed Welding Robot}

The results and analyses of the tensile strength tests of welded mild steel plates of different thickness (see figure 5) using the developed welding robot are shown in Tables 2 to 7.

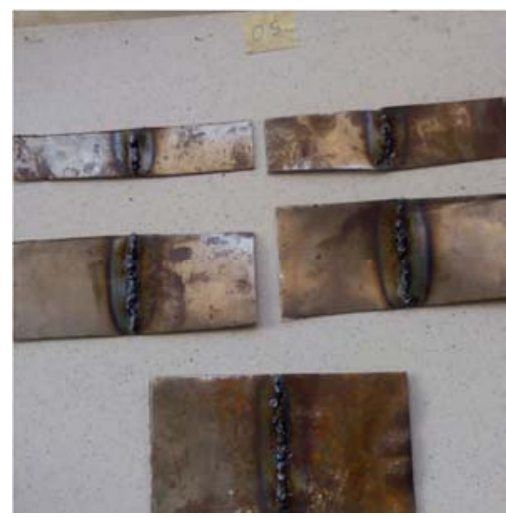

$0.5 \mathrm{~mm}$ Welded Mild Steel Plate Specimen

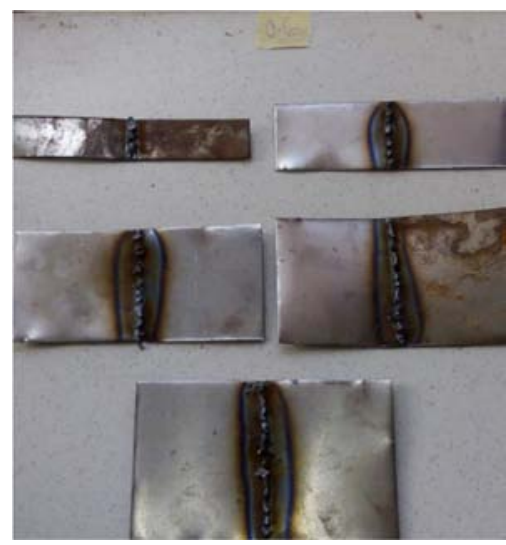

$0.6 \mathrm{~mm}$ Welded Mild Steel Plate Specimen

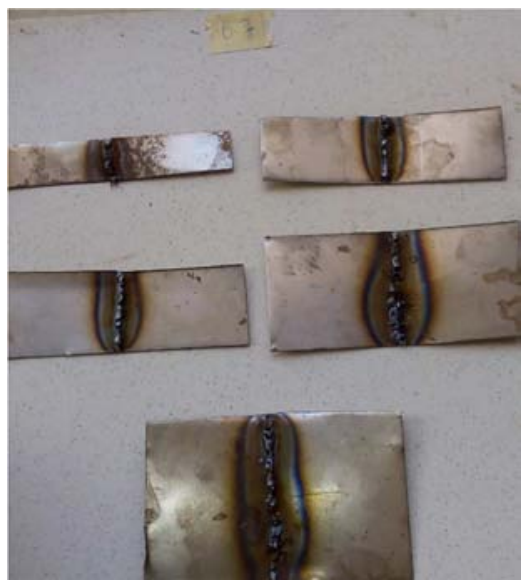

$0.7 \mathrm{~mm}$ Welded Mild Steel Plate Specimen

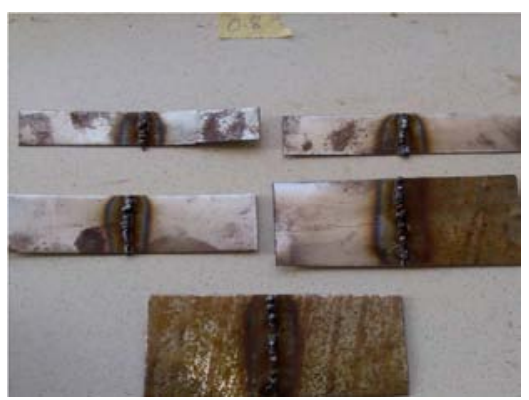

$0.8 \mathrm{~mm}$ Welded Mild Steel Plate Specimen

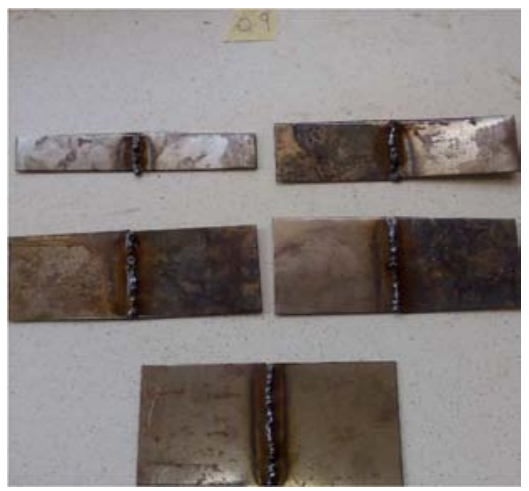

$0.9 \mathrm{~mm}$ Welded Mild Steel Plate Specimen

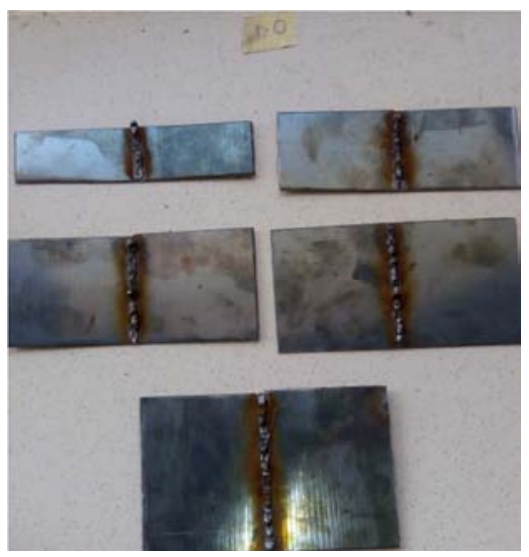

$1.0 \mathrm{~mm}$ Welded Mild Steel Plate Specimen

Figure 5. Welded Mild Steel Plate Specimens using the Developed Welding Robot. 
Table 2. Tensile Test on $0.5 \mathrm{~mm}$ Mild Steel Plate Specimens.

\begin{tabular}{|c|c|c|c|c|c|c|}
\hline $\mathbf{S} / \mathbf{N}$ & $\operatorname{Load}(\mathbf{N})$ & Extension (mm) & Tensile Stress (MPa) & Tensile Strain (mm/mm) & Modulus of Elasticity (MPa) & Break Point (mm) \\
\hline 1 & 1377.23871 & 1.00862 & 58.78099 & 0.01681 & 8695.97473 & 1.17537 \\
\hline 2 & 2104.77915 & 0.70862 & 89.83266 & 0.01181 & 14403.78265 & 1.04181 \\
\hline 3 & 1948.17912 & 1.26694 & 83.14892 & 0.02112 & 8394.52515 & 1.68362 \\
\hline 4 & 1933.04736 & 1.58337 & 82.50309 & 0.02639 & 7966.05911 & 1.74312 \\
\hline 5 & 2892.69276 & 2.01669 & 123.46107 & 0.03361 & 10642.78030 & 2.46700 \\
\hline AVG & 2051.187 & 1.316848 & 87.54535 & 0.021948 & 10020.62 & 1.622184 \\
\hline SD & 487.8069 & 0.453486 & 20.81976 & 0.007558 & 2375.68 & 0.503704 \\
\hline
\end{tabular}

Table 3. Tensile Test on $0.6 \mathrm{~mm}$ Mild Steel Plate Specimens.

\begin{tabular}{|c|c|c|c|c|c|c|}
\hline $\mathbf{S} / \mathbf{N}$ & $\operatorname{Load}(\mathbf{N})$ & Extension (mm) & Tensile Stress (MPa) & Tensile Strain (mm/mm) & Modulus of Elasticity (MPa) & Break Point $(\mathrm{mm})$ \\
\hline 1 & 174.97364 & 1.55862 & 7.46793 & 0.02598 & 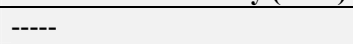 & 3.22531 \\
\hline 2 & 711.03214 & 0.38362 & 30.34708 & 0.00639 & 9394.64111 & 0.58300 \\
\hline 3 & 2886.65723 & 2.10019 & 123.20347 & 0.03500 & 4040.35339 & 2.36637 \\
\hline 4 & 2280.73299 & 0.77531 & 97.34242 & 0.01292 & 23115.09552 & 1.45000 \\
\hline 5 & 811.29335 & 1.04200 & 34.62626 & 0.01737 & 3094.28253 & 1.86662 \\
\hline AVG & 1372.938 & 1.171948 & 58.59743 & 0.019532 & 9911.093 & 1.89826 \\
\hline SD & 1029.963 & 0.601135 & 43.95916 & 0.010019 & 7992.917 & 0.884538 \\
\hline
\end{tabular}

Table 4. Tensile Test on $0.7 \mathrm{~mm}$ Mild Steel Plate Specimens.

\begin{tabular}{|c|c|c|c|c|c|c|}
\hline $\mathbf{S} / \mathbf{N}$ & Load (N) & Extension (mm) & Tensile Stress (MPa) & Tensile Strain (mm/mm) & Modulus of Elasticity (MPa) & Break Point (mm) \\
\hline 1 & 335.41701 & 0.19975 & 14.31571 & 0.00333 & ----- & 0.39944 \\
\hline 2 & 2742.91094 & 0.59181 & 117.06833 & 0.00986 & 19616.97235 & 1.26687 \\
\hline 3 & 569.26035 & 0.67475 & 24.29622 & 0.01125 & 4393.96782 & 0.90512 \\
\hline 4 & 3669.73765 & 1.07512 & 156.62559 & 0.01792 & 12870.72144 & 1.26194 \\
\hline 5 & 5548.74055 & 2.54200 & 236.82205 & 0.04237 & 14355.67932 & 3.15869 \\
\hline AVG & 2573.213 & 1.016686 & 109.8256 & 0.016946 & 12809.34 & 1.398412 \\
\hline SD & 1954.916 & 0.811766 & 83.43645 & 0.013531 & 5467.113 & 0.935499 \\
\hline
\end{tabular}

Table 5. Tensile Test on $0.8 \mathrm{~mm}$ Mild Steel Plate Specimens.

\begin{tabular}{|c|c|c|c|c|c|c|}
\hline $\mathbf{S} / \mathbf{N}$ & Load (N) & Extension (mm) & Tensile Stress (MPa) & Tensile Strain $(\mathrm{mm} / \mathbf{m m})$ & Modulus of Elasticity (MPa) & Break Point (mm) \\
\hline 1 & 3960.70220 & 1.42519 & 169.04405 & 0.02375 & 15964.16626 & 2.68337 \\
\hline 2 & 2921.90202 & 1.05025 & 124.70773 & 0.01750 & 15081.05621 & 2.37481 \\
\hline 3 & 1662.07906 & 0.72531 & 70.93807 & 0.01209 & 19467.34924 & 0.81644 \\
\hline 4 & 3863.44381 & 1.77519 & 164.89303 & 0.02959 & 7167.07382 & 2.89981 \\
\hline 5 & 3533.40730 & 3.44181 & 150.80697 & 0.05736 & 8273.20557 & 4.41700 \\
\hline AVG & 3188.307 & 1.68355 & 136.078 & 0.028058 & 13190.57 & 2.638286 \\
\hline SD & 845.2031 & 0.947198 & 36.07354 & 0.015786 & 4714.397 & 1.150895 \\
\hline
\end{tabular}

Table 6. Tensile Test on $0.9 \mathrm{~mm}$ Mild Steel Plate Specimens.

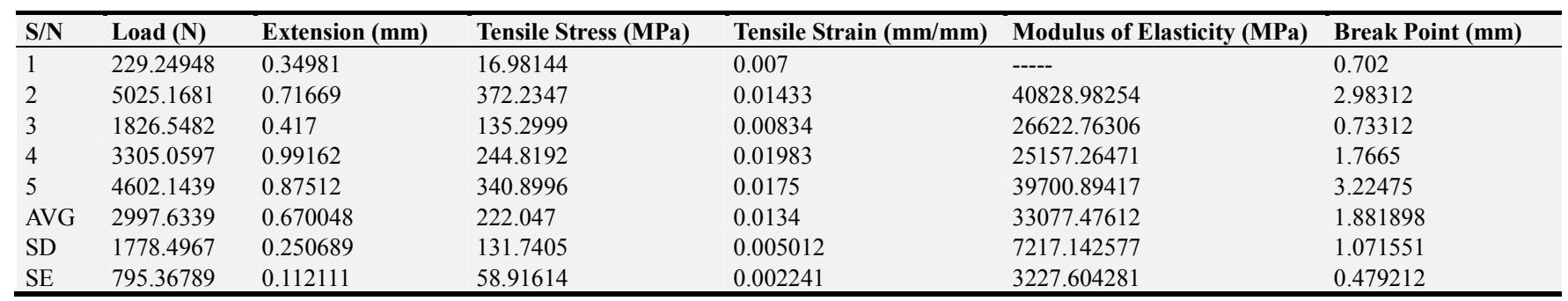

Table 7. Tensile Test on $1.0 \mathrm{~mm}$ Mild Steel Plate Specimens.

\begin{tabular}{|c|c|c|c|c|c|c|}
\hline $\mathbf{S} / \mathbf{N}$ & $\operatorname{Load}(\mathbf{N})$ & Extension (mm) & Tensile Stress (MPa) & Tensile Strain $(\mathrm{mm} / \mathrm{mm})$ & Modulus of Elasticity (MPa) & Break Point (mm) \\
\hline 1 & 4334.66695 & 2.80856 & 185.00499 & 0.04681 & 7275.82855 & 4.66681 \\
\hline 2 & 1310.00392 & 1.03344 & 55.91139 & 0.01722 & 6461.48300 & 1.99987 \\
\hline 3 & 2401.74290 & 1.01700 & 102.50717 & 0.01695 & 14411.11908 & 2.49975 \\
\hline 4 & 4582.03629 & 3.34181 & 195.56279 & 0.05570 & 22870.30334 & 6.86662 \\
\hline 5 & 2560.47770 & 1.05037 & 109.28202 & 0.01751 & 14805.67932 & 1.56669 \\
\hline
\end{tabular}




\begin{tabular}{lllllll}
\hline S/N & Load (N) & Extension $(\mathbf{m m})$ & Tensile Stress (MPa) & Tensile Strain (mm/mm) & Modulus of Elasticity (MPa) & Break Point (mm) \\
\hline AVG & 3037.786 & 1.850236 & 129.6537 & 0.030838 & 13164.88 & 3.519948 \\
SD & 1239.695 & 1.014337 & 52.91057 & 0.016907 & 5967.529 & 1.984045 \\
SE & 554.4083 & 0.453625 & 23.66233 & 0.007561 & 2668.76 & 0.887292 \\
\hline
\end{tabular}

The graphs of the tensile strength test of the welding operation using the developed welding robot for different thicknesses of mild steel plates are shown in Figures 6 to 11.

$0.5: 1,2,3,4,5$

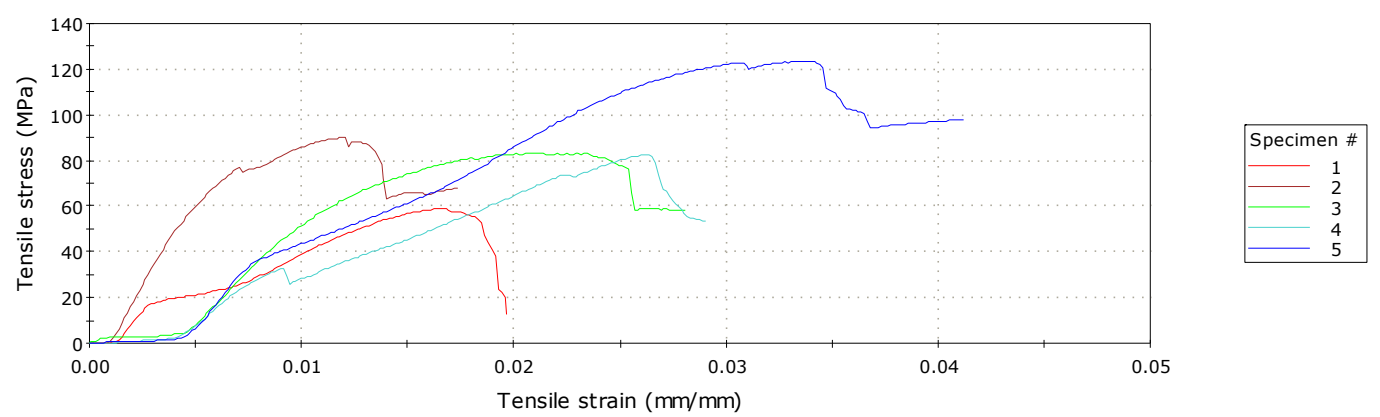

Figure 6. Tensile Test of $0.5 \mathrm{~mm}$ Mild Steel Plate Specimens.

Figure 6 shows initial increases in tensile stress as tensile strain increases; afterwards tensile stress decreases as the tensile strain increases. Sample 5 gave the longest trend of increases in tensile strain followed by 4, 3, 1 and sample 2 gave the shortest trend.

$0.6: 1,2,3,4,5$

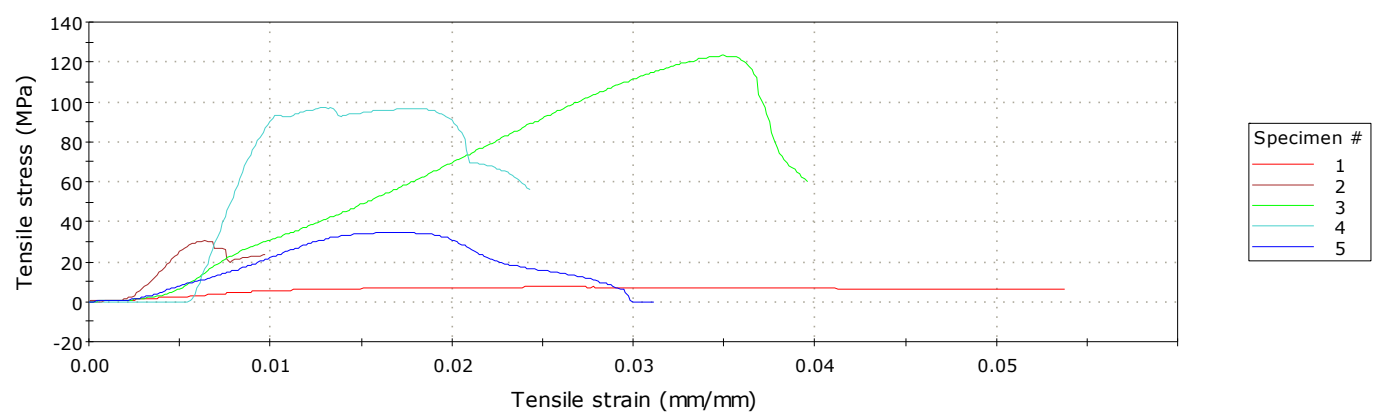

Figure 7. Tensile Test of $0.6 \mathrm{~mm}$ Mild Steel Plate Specimens.

Figure 7 shows increases in tensile stress and tensile strain; later stress decreases as the strain increases. Sample 1 gave the longest trend of increases in tensile strain with constant tensile stress before decreasing, followed by 3 , 5, 4 and sample 2 gives the shortest trend.

$0.7: 1,2,3,4,5$

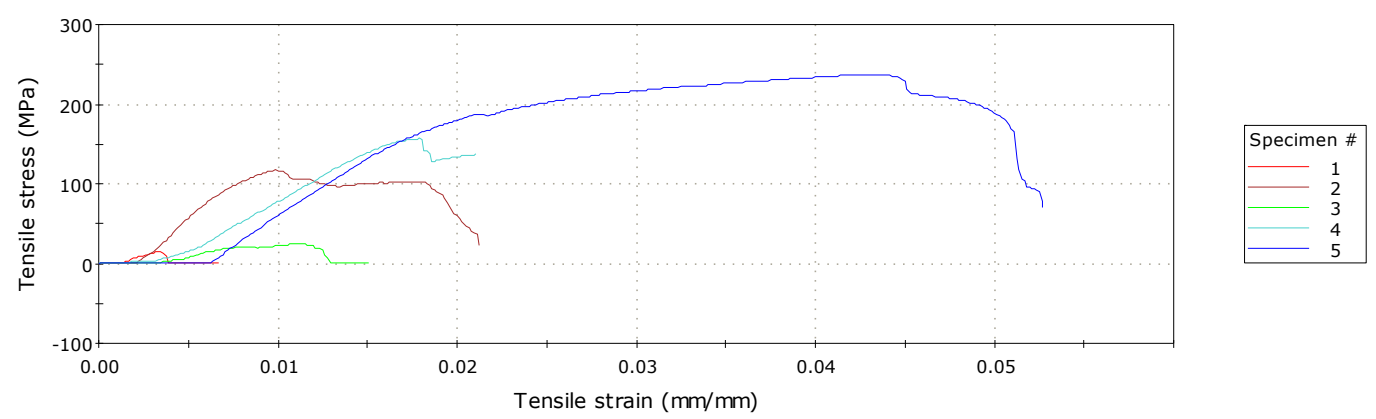

Figure 8. Tensile Test of $0.7 \mathrm{~mm}$ Mild Steel Plate Specimens.

Figure 8 also shows increases in tensile stress and tensile strain; after some time the stress decreases as the strain increases. Sample 5 gave the longest trend of increases in tensile strain followed by 4, 2, 3 and sample 1 gave the shortest trend. 
$0.8: 1,2,3,4,5$

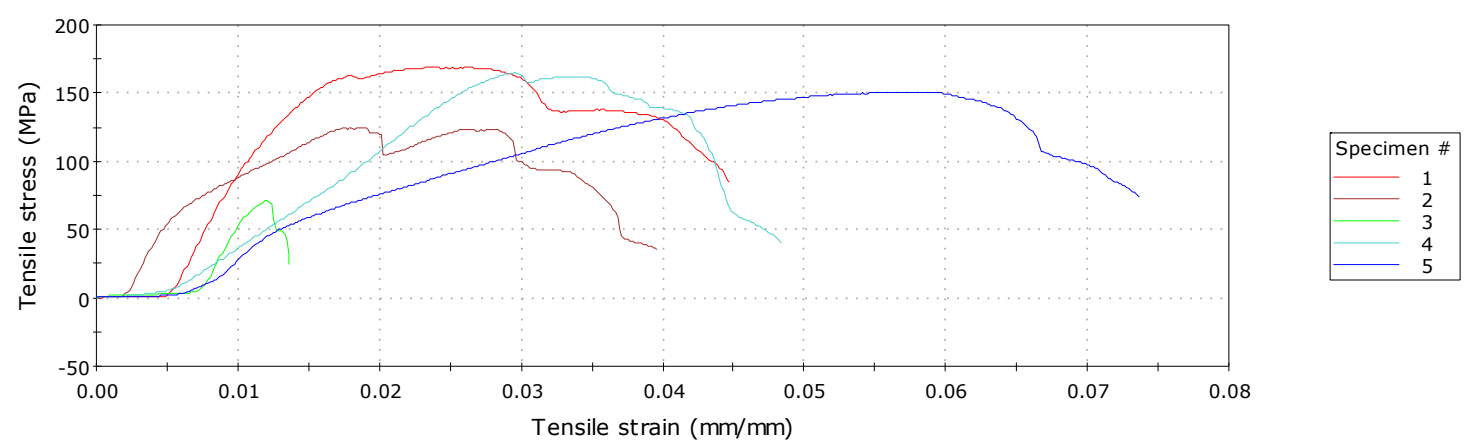

Figure 9. Tensile Test of $0.8 \mathrm{~mm}$ Mild Steel Plate Specimens.

Figure 9 shows increases in both tensile stress and tensile strain; it got to a point where the stress decreases as the strain increases. Sample 5 gave the longest trend of increases in tensile strain followed by 4, 1, 2 and sample 3 gave the shortest trend.

$0.9: 1,2,3,4,5$

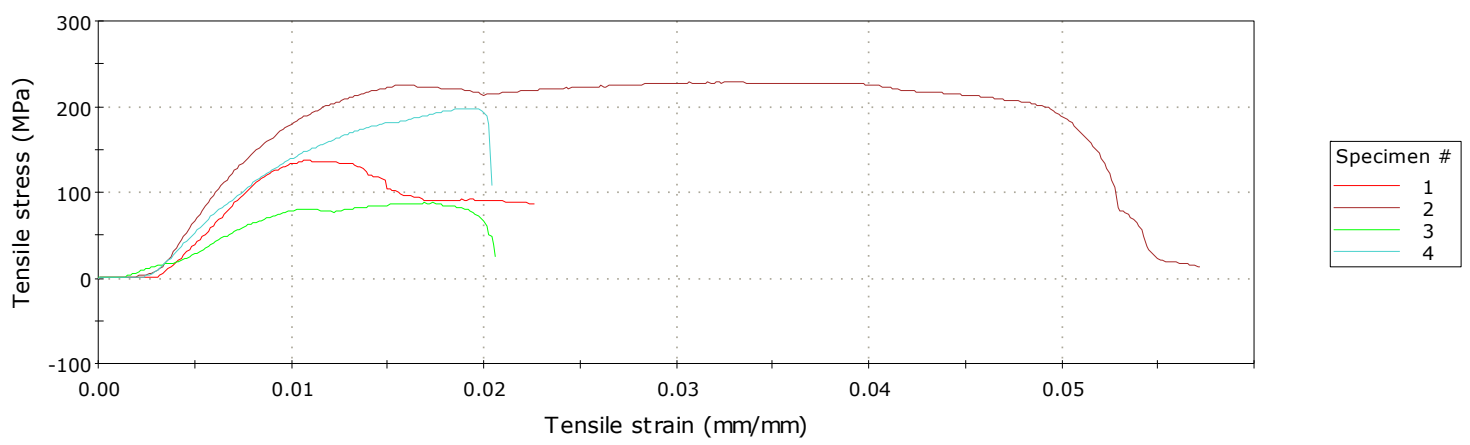

Figure 10. Tensile Test of $0.9 \mathrm{~mm}$ Mild Steel Plate Specimens.

Figure 10 shows increases in tensile stress and tensile strain; the trend changed when stress decreases as the strain increases. Sample 5 gave the longest trend of increases in tensile strain followed by 2, 4, 3 and sample 1 gave the shortest trend

$1 \mathrm{~mm}: 1,2,3,4,5$

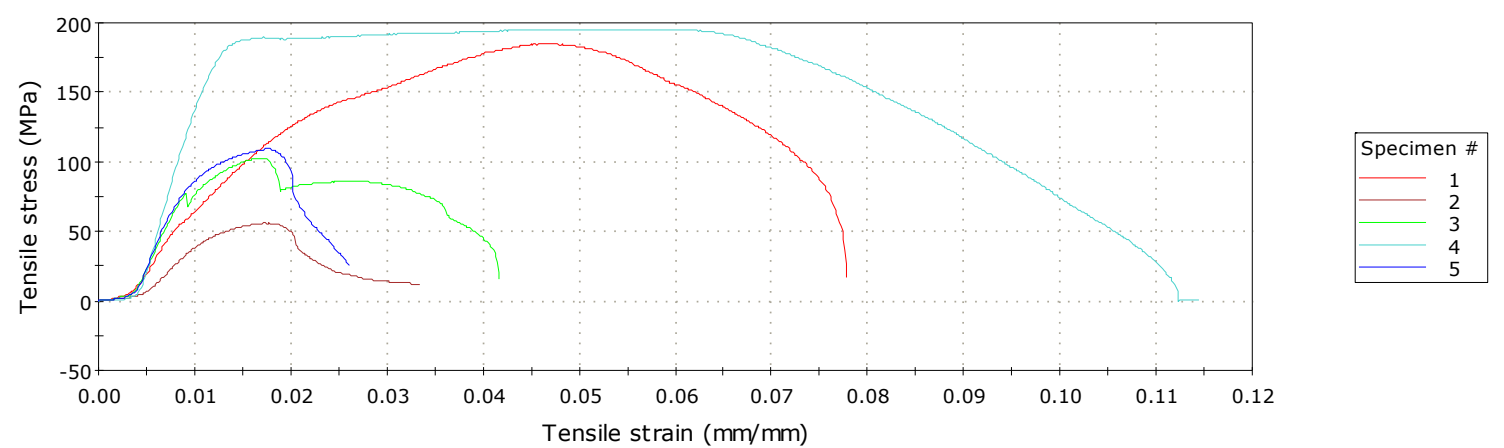

Figure 11. Tensile Test of $1.0 \mathrm{~mm}$ Mild Steel Plate Specimens.

Figure 11 shows increases in tensile stress and tensile strain at first; later stress decreases as the strain increases. Sample 4 gave the longest trend of increases in tensile strain followed by 1, 3, 2 and sample 5 gave the shortest trend.

\subsection{Hardness Test of Welded Mild Steel Plate with the Developed Welding Robot}

The results and analyses of the hardness tests of welded mild steel plates for different gauges using the developed welding robot are shown in Table 8. 
Table 8. Hardness Test on Welded Mild Plates using Developed Welding Robot.

\begin{tabular}{|c|c|c|c|c|c|}
\hline \multicolumn{6}{|c|}{ Hardness Test on the Developed Welding Robot } \\
\hline \multirow{2}{*}{ Number of Test } & \multicolumn{5}{|c|}{ Samples } \\
\hline & A & B & $\mathbf{C}$ & D & $\mathbf{E}$ \\
\hline \multicolumn{6}{|c|}{$0.5 \mathrm{~mm}$ Mild Steel Plate Specimen } \\
\hline 1 & 147 & 149 & 146 & 158 & 157 \\
\hline 2 & 143 & 146 & 143 & 156 & 156 \\
\hline 3 & 143 & 143 & 143 & 143 & 131 \\
\hline 4 & 131 & 143 & 143 & 143 & 128 \\
\hline 5 & 121 & 144 & 131 & 131 & 121 \\
\hline 6 & 118 & 143 & 128 & 143 & 143 \\
\hline AVG & 133.83 & 144.67 & 139.00 & 145.67 & 139.33 \\
\hline SD & 11.29 & 2.21 & 6.86 & 9.09 & 13.77 \\
\hline SE & 4.61 & 0.90 & 2.80 & 3.71 & 5.62 \\
\hline \multicolumn{6}{|c|}{0.6 mm Mild Steel Plate Specim } \\
\hline 1 & 111 & 95.5 & 147 & 95.5 & 111 \\
\hline 2 & 143 & 111 & 143 & 143 & 121 \\
\hline 3 & 143 & 121 & 111 & 111 & 95.5 \\
\hline 4 & 111 & 103 & 103 & 103 & 95.5 \\
\hline 5 & 111 & 111 & 103 & 111 & 95.5 \\
\hline 6 & 95.5 & 95.5 & 95.5 & 103 & 94.3 \\
\hline AVG & 119.08 & 106.17 & 117.08 & 111.08 & 102.13 \\
\hline SD & 17.78 & 9.17 & 20.27 & 15.23 & 10.23 \\
\hline $\mathrm{SE}$ & 7.26 & 3.74 & 8.28 & 6.22 & 4.18 \\
\hline \multicolumn{6}{|c|}{0.7 mm Mild Steel Plate Specimen } \\
\hline 1 & 135 & 137 & 120 & 145 & 116 \\
\hline 2 & 131 & 131 & 111 & 143 & 111 \\
\hline 3 & 121 & 95.5 & 95.5 & 95.5 & 111 \\
\hline 4 & 103 & 95.5 & 95.5 & 95.5 & 111 \\
\hline 5 & 95.5 & 95.5 & 95.5 & 94.3 & 103 \\
\hline 6 & 94.2 & 95.5 & 103 & 94.1 & 102 \\
\hline AVG & 113.28 & 108.33 & 103.42 & 111.23 & 109.00 \\
\hline SD & 16.49 & 18.23 & 9.32 & 23.18 & 4.93 \\
\hline SE & 6.73 & 7.44 & 3.80 & 9.46 & 2.01 \\
\hline \multicolumn{6}{|c|}{$0.8 \mathrm{~mm}$ Mild Steel Plate Specimen } \\
\hline 1 & 111 & 115 & 95.5 & 103 & 95.5 \\
\hline 2 & 121 & 111 & 121 & 103 & 95.5 \\
\hline 3 & 95.5 & 111 & 131 & 95.5 & 95.6 \\
\hline 4 & 95.5 & 103 & 95.5 & 111 & 95.5 \\
\hline 5 & 121 & 95.5 & 95.5 & 121 & 94.5 \\
\hline 6 & 95.5 & 95.5 & 95.5 & 95.5 & 94.3 \\
\hline AVG & 106.58 & 105.17 & 105.67 & 104.83 & 95.15 \\
\hline SD & 11.57 & 7.71 & 14.66 & 8.95 & 0.53 \\
\hline SE & 4.72 & 3.15 & 5.99 & 3.65 & 0.22 \\
\hline \multicolumn{6}{|c|}{$0.9 \mathrm{~mm}$ Mild Steel Plate Specimen } \\
\hline 1 & 102 & 111 & & 137 & 117 \\
\hline 2 & 96.5 & 103 & & 131 & 111 \\
\hline 3 & 95.5 & 95.5 & 95.5 & 111 & 103 \\
\hline 4 & 95.5 & 94.7 & 95.5 & 103 & 103 \\
\hline 5 & 95.7 & 94.8 & 95.5 & 103 & 121 \\
\hline 6 & 95.5 & 94.5 & 103 & 95.5 & 121 \\
\hline AVG & 96.78 & 98.92 & 97.38 & 113.42 & 112.67 \\
\hline SD & 2.36 & 6.17 & 3.25 & 15.33 & 7.61 \\
\hline SE & 0.96 & 2.52 & 1.33 & 6.26 & 3.11 \\
\hline \multicolumn{6}{|c|}{$1.0 \mathrm{~mm}$ Mild Steel Plate Specimen } \\
\hline 1 & 143 & 144 & 111 & 146 & 108 \\
\hline 2 & 143 & 143 & 143 & 143 & 103 \\
\hline 3 & 131 & 121 & 121 & 143 & 121 \\
\hline 4 & 103 & 103 & 121 & 156 & 121 \\
\hline 5 & 95.5 & 121 & 121 & 143 & 131 \\
\hline 6 & 103 & 111 & 95.5 & 95.5 & 121 \\
\hline AVG & 119.75 & 123.83 & 118.75 & 137.75 & 117.50 \\
\hline SD & 19.82 & 15.21 & 14.16 & 19.45 & 9.31 \\
\hline SE & 8.09 & 6.21 & 5.78 & 7.94 & 3.80 \\
\hline
\end{tabular}

Table 8 shows the minimum average hardness values for various mini-robot welded mild steel plates of thickness 0.5 $\mathrm{mm}, 0.6 \mathrm{~mm}, 0.7 \mathrm{~mm}, 0.8 \mathrm{~mm}, 0.9 \mathrm{~mm}$ and $1.0 \mathrm{~mm}$ as $133.83,102.13,103.42,95.15,96.78$ and 117.50 respectively. 
The maximum average hardness values for $0.5 \mathrm{~mm}, 0.6 \mathrm{~mm}$, $0.7 \mathrm{~mm}, 0.8 \mathrm{~mm}, 0.9 \mathrm{~mm}$ and $1.0 \mathrm{~mm}$ thick welded plates were given as $145.67,119.08,113.28,106.58,113.42$ and 137.75 respectively. The research results showed that the developed robot welding samples have high hardness values. This is responsible for low tensile stress values which may also imply low extension of the mini-robot welded samples. The developed robot welding samples gave low values of tensile strain and this was expected since developed robot welding samples had high hardness, low extension and low tensile stress values.

\subsection{Microstructure of Welded Mild Steel Plate with the Developed Welding Robot}

The microstructures of the welded specimen using the developed welding robot for different thickness of mild steel plate are shown in figures 12 to 17 . Five (5) specimen of the size $0.5 \mathrm{~mm}, 0.6 \mathrm{~mm}, 0.7 \mathrm{~mm}, 0.8 \mathrm{~mm}, 0.9 \mathrm{~mm}$ and $1.0 \mathrm{~mm}$ respectively were taken for the experiment as shown in plate 5.

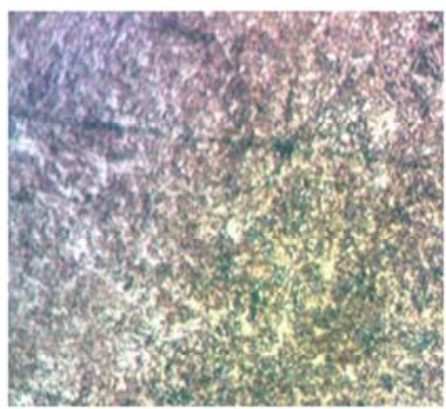

Microstructure on $0.5 \mathrm{~mm}$ Specimen 1

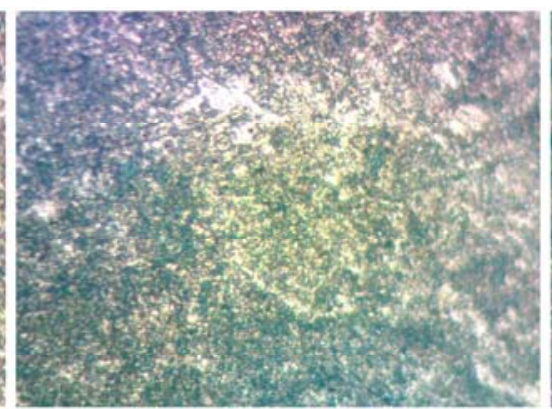

Microstructure on $0.5 \mathrm{~mm}$ Specimen 2

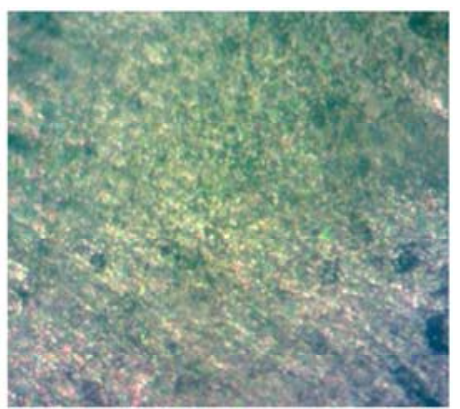

Microstructure on $0.5 \mathrm{~mm}$ Specimen 3

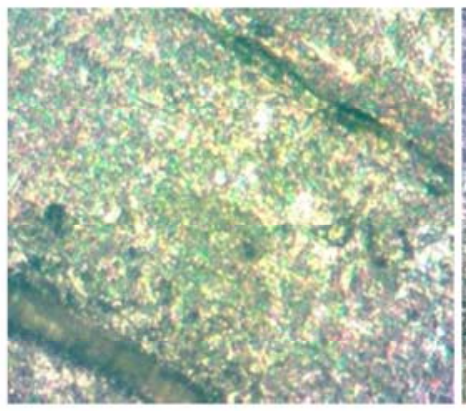

Microstructure on $0.5 \mathrm{~mm}$ Specimen 4

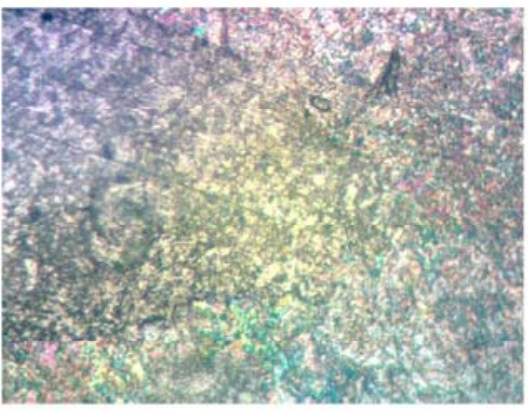

Microstructure on $0.5 \mathrm{~mm}$ Specimen 5

Figure 12. Microstructure of $0.5 \mathrm{~mm}$ Welded Specimens for the Developed Welding Robot.

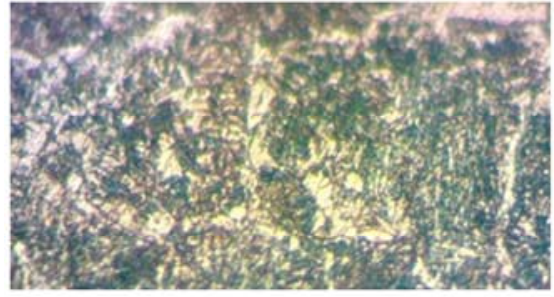

Microstructure on $0.6 \mathrm{~mm}$ Specimen 1

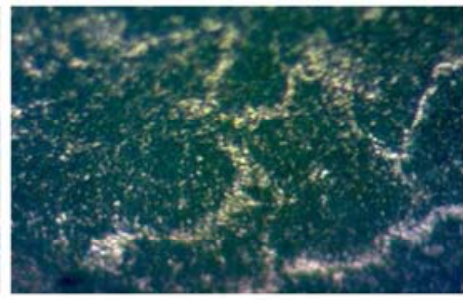

Microstructure on $0.6 \mathrm{~mm}$ Specimen 2

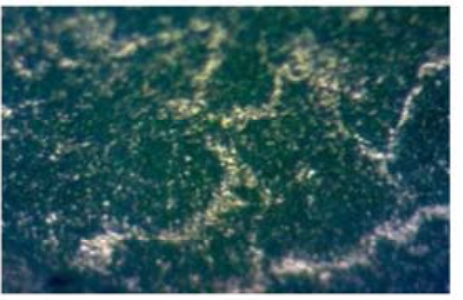

Microstructure on $0.6 \mathrm{~mm}$ Specimen 3
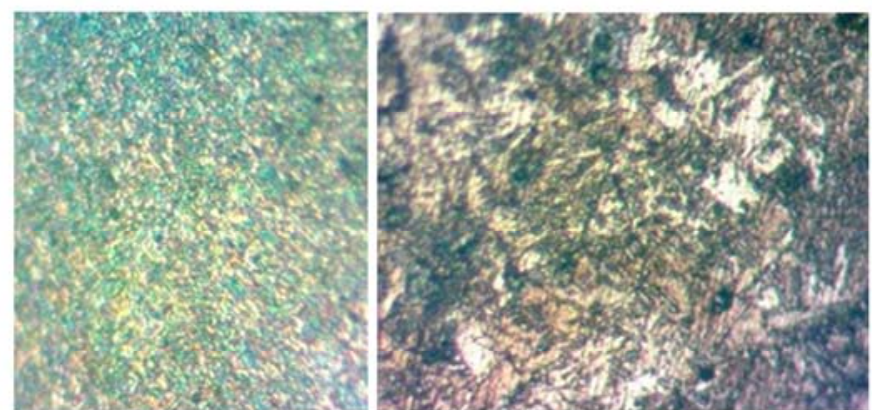

Microstructure on $0.6 \mathrm{~mm}$ Specimen 4 Microstructure on $0.6 \mathrm{~mm}$ Specimen 5

Figure 13. Microstructure of $0.6 \mathrm{~mm}$ Welded Specimens for the Developed Welding Robot. 


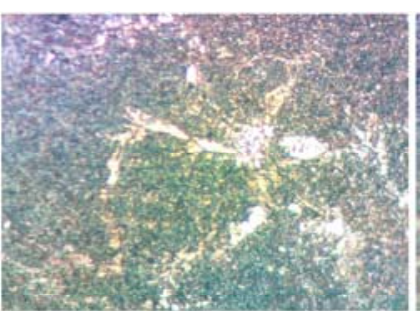

Microstructure on $0.7 \mathrm{~mm}$ Specimen 1

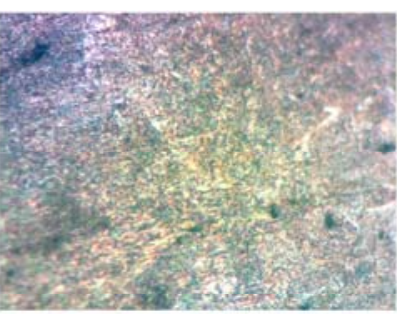

Microstructure on $0.7 \mathrm{~mm}$ Specimen 2

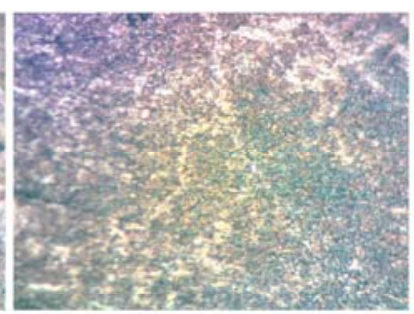

Microstructure on $0.7 \mathrm{~mm}$ Specimen 3

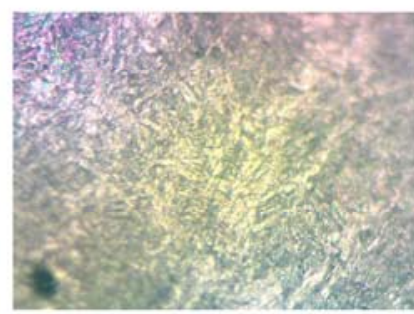

Microstructure on $0.7 \mathrm{~mm}$ Specimen 4

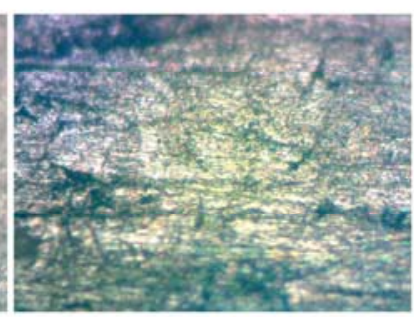

Microstructure on $0.7 \mathrm{~mm}$ Specimen 5

Figure 14. Microstructure of $0.7 \mathrm{~mm}$ Welded Specimens for the Developed Welding Robot.

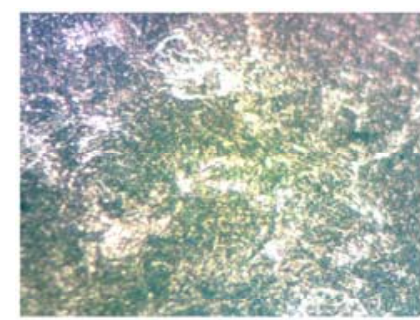

Microstructure on $0.8 \mathrm{~mm}$ Specimen 1

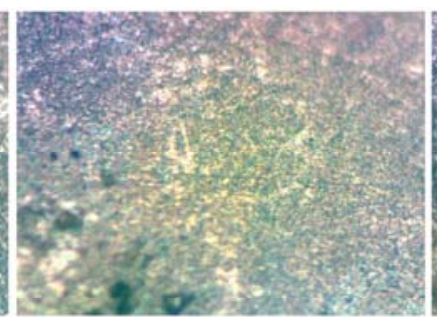

Microstructure on $0.8 \mathrm{~mm}$ Specimen 2

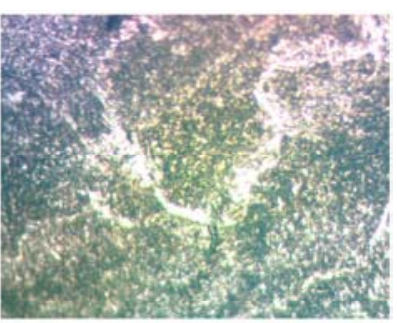

Microstructure on $0.8 \mathrm{~mm}$ Specimen 3

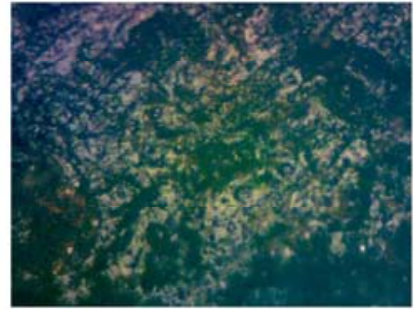

Microstructure on $0.8 \mathrm{~mm}$ Specimen 4

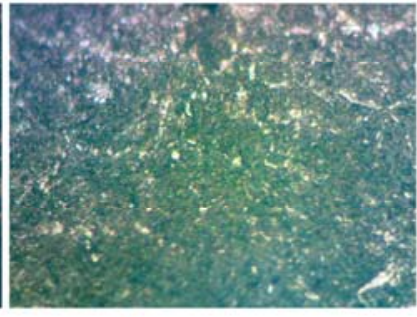

Microstructure on $0.8 \mathrm{~mm}$ Specimen 5

Figure 15. Microstructure of $0.8 \mathrm{~mm}$ Welded Specimens for the Developed Welding Robot.

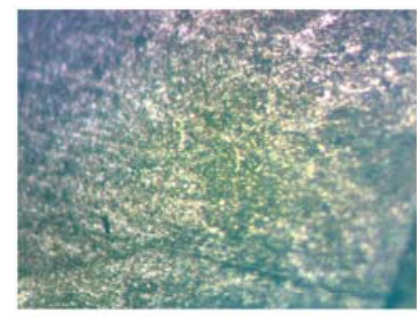

Microstructure on $0.9 \mathrm{~mm}$ Specimen 1

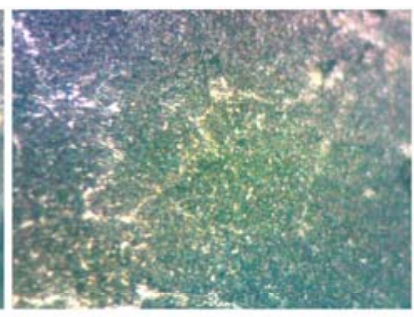

Microstructure on $0.9 \mathrm{~mm}$ Specimen 2

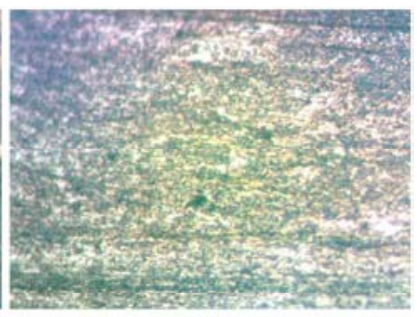

Microstructure on $0.9 \mathrm{~mm}$ Specimen 3

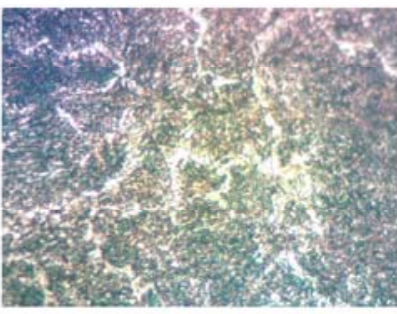

Microstructure on $0.9 \mathrm{~mm}$ Specimen 4

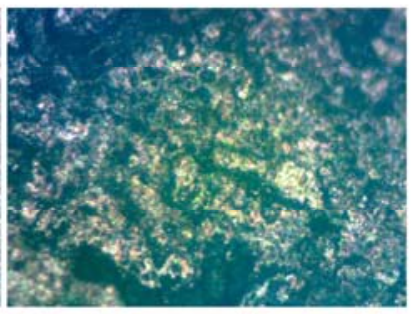

Microstructure on $0.9 \mathrm{~mm}$ Specimen 5

Figure 16. Microstructure of $0.9 \mathrm{~mm}$ Welded Specimens for the Developed Welding Robot. 


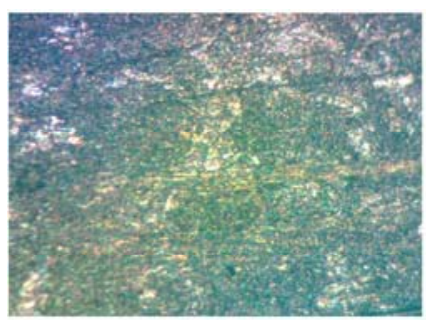

Microstructure on $1.0 \mathrm{~mm}$ Specimen 1

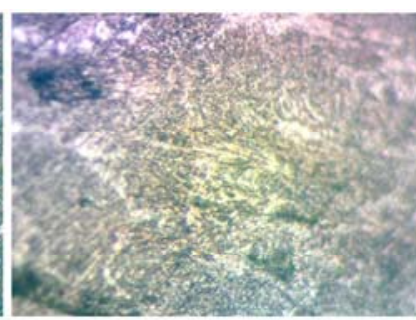

Microstructure on $1.0 \mathrm{~mm}$ Specimen 2

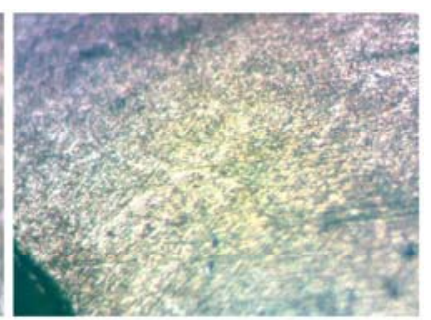

Microstructure on $1.0 \mathrm{~mm}$ Specimen 3

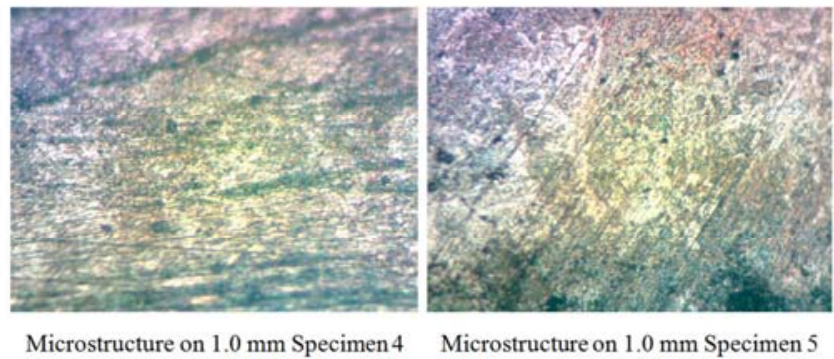

Figure 17. Microstructure of $1.0 \mathrm{~mm}$ Welded Specimens for the Developed Welding Robot.

\subsection{Discussion of Microstructural Analysis Results}

The microstructure investigation of the welded mild steel plates has been performed by the help of an optical microscope. The optical microscope with an interfaced computer in which microstructure study was done is shown in the figure 3 . There is more pearlite $\left(\alpha+\mathrm{Fe}_{3} \mathrm{C}\right)$ than ferrite $(\alpha)$ in the microstructural test of the welded materials using developed welding robot as shown in figures 12 to 17 . This indicates that the weldment portion is hard. The high hardness and high strength and decrease in porosity of the weld bead are caused by the fine grain in the microstructure.

\section{Conclusion}

The mechanical and microstructural properties of the welded joints have been discussed. The results shows the minimum average hardness values for various mini-robot welded mild steel plates of thickness $0.5 \mathrm{~mm}, 0.6 \mathrm{~mm}, 0.7$ $\mathrm{mm}, 0.8 \mathrm{~mm}, 0.9 \mathrm{~mm}$ and $1.0 \mathrm{~mm}$ as $133.83,102.13,103.42$, $95.15,96.78$ and 117.50 while the maximum average hardness values were determined as $145.67,119.08,113.28$, $106.58,113.42$ and 137.75 respectively. The research results showed that the developed robot welding samples have high hardness values. This is responsible for low tensile stress values which may also imply low extension of the mini-robot welded samples. The developed robot welding samples gave low values of tensile strain and this was expected since developed robot welding samples had high hardness, low extension and low tensile stress values.

The microstructural analysis showed that the mini-robot welded samples have more of fine structure than coarse structure (that is more pearlite than ferrite). The developed welding robot has also presented, from experimentation, significantly less welding time, high length of weld and hence, a high range of welding speed. The developed welding robot has worked very well and presented quality welds from the results of Microstructural Analyses.

\section{References}

[1] Charde, N. (2012a). Characterization of spot weld growth on dissimilar joints with different thicknesses. Journal of Mechanical Engineering and Sciences, 2, 172-180.

[2] Charde, N. (2012b). Effects of electrode deformation of resistance spot welding on 304 austenitic stainless steel weld geometry. Journal of Mechanical Engineering and Sciences, 3, 261-270.

[3] Charde, N. (2013). Microstructure and fatigue properties of dissimilar spot welds joints of aisi 304 and aisi 1008 . International Journal of Automotive and Mechanical Engineering, 7, 882-899.

[4] Kukiełka, L. (1989). Designating the field areas for the contact of a rotary burnishing element with the rough surface of a part, providing a high-quality product. Journal of Mechanical Working Technology, 19, 319-356.

[5] Shah, L. H., Akhtar, Z., \& Ishak, M. (2013). Investigation of aluminum-stainless steel dissimilar weld quality using different filler metals. International Journal of Automotive and Mechanical Engineering, 8, 1121-1131.

[6] Lopez-Juarez, I., Rios-Cabrera, R., \& Davila-Rios, I. (2010). Implementation of an intelligent robotized gmaw welding cell, part 2: Intuitive visual programming tool for trajectory learning.

[7] Rafiqul, M. I., Ishak, M., \& Rahman, M. M. (2012). Effects of heat input on mechanical properties of metal inert gas welded $1.6 \mathrm{~mm}$ thick galvanized steel sheet. IOP Conference Series:Materials Science and Engineering, 36 (1).

[8] Rahman, M. M., Arrifin, A. K., Nor, M. J. M., \& Abdullah, S. (2008). Fatigue analysis of spot-welded joint for automative structures. SDHM Structural Durability and Health Monitoring, 4 (3), 173-180.

[9] Rahman, M. M., Bakar, R. A., Noor, M. M., Rejab, M. R. M., \& Sani, M. S. M. (2008). Fatigue life prediction of spotwelded structures: A finite element analysis approach European Journal of Scientific Research (Vol. 22, pp. 444456). 
[10] Rahman, M. M., Rosli, A. B., Noor, M. M., Sani, M. S. M., \& Julie, J. M. (2009). Effects of spot diameter and sheets thickness on fatigue life of spot welded structure based on fea approach. American Journal of Applied Sciences, 6 (1), 137142.

[11] Yang, W. H., \& Tarng, Y. S. (1989). Design optimization of cutting parameters for turning operations based on the taguchi method. Journal Material Processing Technology, 84, 122129.

[12] Talabi, S. I., Owolabi, O. B., Adebisi, J. A., Yahaya, T. (2014). Effect of welding variables on mechanical properties of low carbon steel welded joint. Advances in Production Engineering \& Management. 9 (4): 181-186.

[13] Didit Sumardiyanto and Sri Endah Susilowati (2019). Effect of Welding Parameters on Mechanical Properties of Low Carbon Steel API 5L Shielded Metal Arc Welds, American Journal of Materials Science, 9 (1): 15-21

[14] Manning, R., Ewing, J. (2009). RACQ Vehicles Technologies. (2009). Temperatures in cars survey. RACQ Vehicles Technologies, 1-21.

[15] Greyjevo, O. G. T. V. Z., \& Metodo, A. I. T. (2009). Optimization of weld bead geometry in tig welding process using grey relation analysis and taguchi method. Materiali in tehnologije, 43 (3), 143-149.
[16] Oladebeye D. H, 2Adejuyigbe S. B., 3Kareem B (2020). Predictive Model Development for Welding Mini-robot, International Journal of Engineering Development and Research, 8 (2): 275-288.

[17] Oladebeye D. H., 2Adejuyigbe S. B., 3Ayodeji S. P (2020). Comparative Analysis of Mechanical Properties of Mild Steel Plates Welded with the Developed Welding Robot and Manual Electric Arc Welding, International Journal of Engineering Development and Research, 8 (2): 289-302.

[18] Nuraini1, A. A., Zaina, A. S. and Azmah Hanim, M. A. (2014). The effects of welding parameters on butt joints using robotic gas metal arc welding, Journal of Mechanical Engineering and Sciences (JMES), Vol. 6, pp. 988-994.

[19] Sumardiyanto, D., Susilowati, S. E., \& Cahyo, A. (2018). Effect of Cutting Parameter on Surface Roughness Carbon Steel S45C. Journal of Mechanical Engineering and Automation, 8 (1), 1-6.

[20] Maksuti, R. (2016). Impact of the Acicular Ferrite on the Charpy V-Notch Toughness of Submerged Arc Weld Metal Deposits. International Journal of Scientific \& Engineering Research, 7 (8): 1149-1155. 